\title{
Magnetic field induced strain assisted by stress in Ni-Fe-Ga- Co single crystals
}

\author{
F. Masdeu, J. Pons, E. Cesari, S. Kustov, J. Dutkiewicz*, Y. Chumlyakov** \\ Dept. de Física, Univ. de les Illes Balears, Palma de Mallorca, Spain \\ *Institute of Metallurgy and Material Science of the Polish Academy of Sciences, Kraków, Poland \\ **Siberian Physical-Technical Institute, Tomsk, Russia
}

\begin{abstract}
:
Ferromagnetic shape memory alloys (FSMA) have the possibility to induced a strain by applying a magnetic field. The main advantage of the FSMA is that the strain cycling frequency is two orders of magnitude higher than coventional shape memory alloys. The best alloy showing this effect is the Ni-Mn-Ga system, with a high mobility of its martensite variants and high magnetocrystalline anisotropy constant. Nevertheless, due to the high brittleness of this alloy, other systems (Ni-Fe-Ga, $\mathrm{Co}-\mathrm{Ni}-\mathrm{Al}, \mathrm{Co}-\mathrm{Ni}-\mathrm{Ga}, . .$.$) are being investigated as an alternative to Ni-Mn-Ga.$

In the current work, Ni-Fe-Ga-Co single crystals have been studied. In spite of the formation of L10 martensite (low mobility of the variants), the [001] crystals exhibited magnetic-field-induced strains (in tension) larger than $2 \%$, under an assisting tensile stress around $16 \mathrm{MPa}$ and fields below $15 \mathrm{kOe}$. In martensitic samples previously compressed, application of a constant tensile stress along the same axis together with a perpendicular magnetic field produces the elongation of the sample by variant reorientation, as one of the variants rotates its c axis from the field direction to the stress-axis direction. An estimated magnetostress of $\sim 0.8 \mathrm{MPa}$ is in good agreement with the theoretical value given by the ratio of magnetocrystalline anisotropy constant and twinning shear.
\end{abstract}

Keywords: Ferromagnetic shape memory alloys, magnetic-field-induced strain 